\title{
BIQ-BYWORD
}

\section{Effects of Fresh Royal Jelly on the Proliferation of Human Hepatoma Cell}

\section{Line SMMC-7721}

Li Wei, Wu Wei, Wang Mingmin

Qingdao No.6 People's Hospital

\section{ARTICLE INFO}

Article history:

Published online: $30^{\text {th }}$ Sept 2017

Key words:

fresh royal jelly

human hepatocellular carcinoma

cell line SMMC-7721

cell proliferation

inhibitory effect

\section{ABSTRACT}

Objective: To investigate the effect of fresh royal jelly on the proliferation of human hepatoma cell line SMMC-7721. Methods: We found that the administration of fresh royal jelly could alleviate the condition of hepatocellular carcinoma patients in a certain extent. The human hepatocellular carcinoma cell line SMMC-7721 was cultured in vitro. MTT colorimetric method was used to treat fresh cells and serum containing human serum. The effect of SMMC-7721 proliferation was observed. Results: The aqueous solution of fresh royal jelly had a certain effect on the proliferation of hepatoma cell line SMMC-7721 in a dose-dependent manner. The serum containing fresh royal jelly could inhibit the proliferation of human hepatoma cell line SMMC-7721, and its inhibitory effect showed dose-dependent. Conclusion: The serum containing fresh royal jelly has a significant inhibitory effect on the proliferation of human hepatoma cell line SMMC-7721 and its anti-cancer effect may be derived from its metabolites or stimulating the formation of immune-reactive substances in the body, in which in the clinical treatment of liver cancer and research have a certain value.

Corresponding author:

Wang Mingmin, Qingdao No.6 People's Hospital, No.9, Fushun Road, Shibei District,

Qingdao City, Shandong, China, wangmm1963@sina.com 


\section{Introduction}

Hepatocellular carcinoma is one of the most common malignancies in the world. The data show that it is in the 6th place in the global malignancy. It is ranked 5th in China and the second in the world and the death of malignant tumor in China. And the mortality rate accounts for about half of the world. Although China through the injection of hepatitis $B$ vaccine shown a significant reduction in the rate of hepatitis B virus infection and antiviral treatment slowed the progress of hepatitis B, hepatitis $\mathrm{C}$ and cirrhosis patients to a certain extent, so that the occurrence of liver cancer and mortality decreased significantly, minimally invasive. Timely surgery to extend the survival time of some patients with liver cancer, molecular biology research progress was given the new treatment which may be, but still a drop in the bucket, it is still a medical problem, the patient's life killer. Therefore, the use of integrated medical control means of its full intervention is to improve the survival rate of patients to extend the survival time in the most effective way. Royal jelly is mainly the by products from the worker bee pharyngeal gland, palatal gland secretion of a slurry material which contains a variety of active substances and rich in nutrients, such as protein, vitamin A, B2, C, E, trace elements germanium, selenium, more importantly is that it also contains a special unsaturated fatty acid (sunflower acid) and flavonoids, which can regulate of cells and humoral immunity to achieve significant anti-cancer effect, thus it was classified as pure natural health products ${ }^{[1]}$. In recent years, the medicinal value of royal jelly at home and abroad has been widely concerned by the clinical medicine community and gradually applied to cancer patients in the immunotherapy process. We investigated the effects of royal jelly and in vitro on the proliferation of human hepatocellular carcinoma cell line SMMC-7721 in order to explore its mechanism. The results are reported as follows.

\section{Materials and method}

\subsection{Experimental material}

The human hepatocellular carcinoma cell line SMMC-7721 was purchased from Shanghai Jihe Biotechnology Co., Ltd. and RPMI1640 medium was provided by Beijing Suolai Bao Technology Co., Ltd. and the newborn calf serum was purchased from Zhejiang Tianhang Biotechnology Co., Ltd. Co., Ltd. Fresh royal jelly by the Shaanxi Tektronix Biotechnology Co., Ltd., batch number 047232, tetramethyl azo blue and trypsin were purchased from Shanghai Shuji Biological Technology Co., Ltd., UV visible microplate detector model Veritas by the Beijing original Ping Hao Biotechnology Co., Ltd. to provide. In addition, from Nanjing Junke Biological Engineering Co., Ltd. purchased $180 \sim 200$ g male SD rats.

\subsection{Method}

\subsubsection{Preparation of fresh royal jelly aqueous solution}

In serum-free RPMI1640 added fresh royal jelly, the mixture prepared into a $0.2 \mathrm{mg} / \mathrm{ml}$ concentration of the suspension, the use of chemical analysis filter paper filter and then under the action of $0.22 \mathrm{um}$ microporous membrane. The bacteria were filtered and the filtrate was taken and diluted to $0.002,0.02$ and $0.2 \mathrm{mg} / \mathrm{ml}$ for a variety of concentrations.

\subsubsection{Cell proliferation detection method}

By using MTT colorimetric method for cell proliferation detection. In the 96 -well plate, $2 \times 104$ cells $/ \mathrm{ml}$ on single cell suspension, 100ul/well. After confirming the cell growth status, the supernatant was discarded and the appropriate serum-free culture medium was added to keep the serum in a hungry state and the supernatant was discarded. Then, the supernatant was discarded and different concentrations of fresh royal jelly solution containing serum was 
added, so that the serum concentration remained at $10 \%$, fresh royal jelly solution at different concentrations of $0.001,0.01$ and $0.1 \mathrm{mg} / \mathrm{ml} .10 \%$ FBS medium was used as the control group, and cultured for about 48 hours. 0.5\% MTT was added. After incubation for 4 hours, the supernatant was discarded and DMSO150uj was added. The microcrystals were used for the oscillation, in which $10 \mathrm{~min}$ is appropriate. At $490 \mathrm{~nm}$ UV visible microplate detection to determine its absorbance value.

\subsubsection{Preparation of serum}

20 male SD rats were randomly divided into control group and observation group, the control group: given normal saline gavage, observation group: given $0.5 \mathrm{~g} / \mathrm{kg}$ royal jelly, $10 \mathrm{ml} / \mathrm{kg}, 2$ Times $/ \mathrm{d}$, the two groups were fed continuously for 10 days for $12 \mathrm{~h}$ before the last administration of fasting and after $1 \mathrm{~h}$ of administration, given the carotid artery intubation and the implementation of sterile blood collection and collection of blood preservation to $8 \mathrm{~h}$ is appropriate and then given centrifugal treatment, the centrifugal rate remained at $3000 \mathrm{r} / \mathrm{min}$, in which to $10 \mathrm{~min}$ is appropriate for the separation of serum. And then use 0.22 um microporous membrane filter on the bacteria, after packaging in $-20{ }^{\circ} \mathrm{C}$ environment to be preserved. Before use at $56{ }^{\circ} \mathrm{C}$ under the conditions to distinguished fire and in serum-free RPMI1640 medium and diluted into the experimental study of the required concentration, kept for the reservation purposes.

\subsubsection{Detection of Cell Proliferation at Different Concentrations}

The serum concentration was $10 \%, 20 \%$ and $40 \%$ respectively. The concentration of the serum was set in different dose groups. The FBS control group and the serum control group included 6 wells, 100ul / \% Serum concentration of $5 \%$ and $10 \%$ of the two doses of serum;
$20 \%$ serum concentration of serum containing $5 \%, 10 \%$,

$20 \%$ of the three different doses, $40 \%$ of the serum concentrations were $5 \%, 10 \%, 20 \%$ and $40 \%$ of the serum dose of 4 doses. The detection method is the same as 1.2.2. The inhibition rate of cell proliferation was calculated by the formula (1-MS group A mean / BS group A mean) $\times 100 \%$

\subsection{Statistical analysis}

Statistical analysis software is SPSS 22.0, different groups of cell proliferation rate (\%), the comparison between groups using $\mathrm{X}^{2}$ test; different groups of absorbance values related to the use of indicators $\left(\bar{s}_{ \pm}\right)$, and $\mathrm{t}$ test to compare between groups to give statistical analysis to determine the value of $P$ statistically significant. If $\mathrm{P}<0.05$ determine the difference is significant, there is a significant statistical significance, on the contrary, there is no statistical significance.

\section{Results}

\subsection{Effect of aqueous solution of royal jelly on} the proliferation of hepatoma cell line SMMC 721

The results showed that the absorbance at different doses of royal jelly group was significantly higher than that of control group $(\mathrm{P}<0.05)$, which was statistically significant and showed significant dose-dependent, as shown in Table 1.

\subsection{Effect of royal jelly serum on proliferation of hepatocellular carcinoma cell line}

The results showed that serum containing SMMC-7721 could inhibit the proliferation of human hepatoma cell line SMMC-7721, which was significantly higher than that of control group $(\mathrm{P}<0.05)$ and the difference was

Table 1 Effect of royal jelly solution on the proliferation of hepatoma cell line SMMC-721 ( \pm s)

\begin{tabular}{lll} 
Group & Dosage $\mathrm{C} / \mathrm{mg} \cdot \mathrm{ml}^{-1}$ & Absorbance value \\
\hline Control group & - & $0.83 \pm 0.02$ \\
\hline
\end{tabular}




\begin{tabular}{lll}
\hline Royal jelly group & 0.1 & $1.22 \pm 0.03^{*}$ \\
& 0.01 & $1.16 \pm 0.04^{*}$ \\
& 0.001 & $1.12 \pm 0.03^{*}$ \\
\hline
\end{tabular}

Note: Compared with the control group, $* \mathrm{P}<0.05$

statistically significant $(\mathrm{P}<0.05)$. Compared with the calf serum, the effect of blank serum on the cell growth was more significant $(\mathrm{P}<0.05)$

\section{Discussion}

Royal jelly is the food for life of the queen bee, its life is generally $3-4$ years, the elderly up to 8 years, while

Table 2 Effect of royal jelly serum on proliferation of hepatocellular carcinoma cell lines $\left(\overline{\mathbf{x}}_{ \pm} \mathrm{s}\right)$

\begin{tabular}{|c|c|c|c|}
\hline $\begin{array}{c}\text { Serum Concentration } \\
(\%)\end{array}$ & Group & Absorbance value & Inhibition percentage $(\%)$ \\
\hline \multirow[t]{4}{*}{10} & FBS & $0.75 \pm 0.03$ & \\
\hline & BS & $0.98 \pm 0.05^{\wedge}$ & \\
\hline & $5 \% \mathrm{MS}$ & $0.96 \pm 0.02 *$ & 4.76 \\
\hline & $10 \% \mathrm{MS}$ & $0.88 \pm 0.06^{*}$ & 9.63 \\
\hline \multirow[t]{4}{*}{20} & FBS & $0.87 \pm 0.03$ & \\
\hline & BS & $1.03 \pm 0.03^{\wedge}$ & \\
\hline & $5 \% \mathrm{MS}$ & $0.93 \pm 0.02 *$ & 9.32 \\
\hline & $10 \% \mathrm{MS}$ & $0.94 \pm 0.02 *$ & 9.75 \\
\hline \multirow[t]{7}{*}{40} & $20 \% \mathrm{MS}$ & $0.85 \pm 0.04 *$ & 17.52 \\
\hline & FBS & $1.02 \pm 0.02$ & \\
\hline & BS & $1.21 \pm 0.02 *$ & \\
\hline & $5 \% \mathrm{MS}$ & $1.02 \pm 0.03 *$ & 14.03 \\
\hline & $10 \% \mathrm{MS}$ & $1.00 \pm 0.03^{*}$ & 16.10 \\
\hline & $20 \% \mathrm{MS}$ & $0.83 \pm 0.01 *$ & 29.42 \\
\hline & $40 \% \mathrm{MS}$ & $0.73 \pm 0.01 *$ & 35.73 \\
\hline
\end{tabular}

Note: Compared with the BS group, ${ }^{*} \mathrm{P}<0.05$, compared with the FBS group, $\wedge$ P $<0.05$ 
while the ordinary worker bee only taking royal jelly for several days, its life is generally only 50 days, the longest but 8 months, which indicate that royal jelly has a very high nutritional value and anti-oxidation and anti-aging effect. The occurrence of hepatocellular carcinoma has a mechanism of oxidative damage similar to that of aging. Studies have found that royal jelly can accelerate cell and tissue regeneration ${ }^{[2]}$, repair the role of DNA damage. Foreign scholars in the study found that royal jelly in a large number of trace elements selenium, vitamin A and royal jelly acid and other substances, which can inhibit the growth of cancer cells $s^{[3-6]}$, the material part of the water-soluble, partially non-water soluble, the usage of culture in this study to dissolve royal jelly, in vitro its role in the cell is mainly water-soluble components, non-water-soluble components in the presence of tumor suppressor substances which are often removed to participate in the reaction system of the liver cancer cells The inhibitory effect of growth is largely masked ${ }^{[7-8]}$ and the role of serum containing drugs in the body, the mechanism is more complex, which may improve the immune function to a certain extent and activate the body's immune active substances and play an anti-cancer effect. Royal jelly in vitro culture of cancer cells directly, failed to fully participate in the process of the body, its anti-cancer effect failed to play, but to promote the proliferation of cancer cells ${ }^{[9]}$. The results showed that the absorbance values of different doses of royal jelly aqueous solution were significantly higher than those of the control group ( $\mathrm{P}<0.05)$, which was statistically significant and showed significant dose-dependent. The serum of the rat serum was significantly higher than that of the control group (P $<0.05$ ), and the serum level of SMMC-7721 was significantly higher than that of the control group ${ }^{[10]}$. Cell growth promotion was more significant, the difference was statistically significant $(\mathrm{P}<0.05)$. In conclusion, the aqueous solution of royal jelly has a certain effect on the proliferation of hepatocarcinoma cell line SMMC-7721 in vitro, and the drug-containing serum has a significant inhibitory effect on the proliferation of hepatoma cell line SMMC-7721, which is consistent with the related domestic and foreign studies. In other words, promote the therapeutic value of royal jelly on liver cancer.

\section{References}

[1] Qian Jian Sheng, Li Yu, Dou Ji Wei. Effects of miR-26a on Proliferation and Migration of Human Hepatocellular Carcinoma Cell Line SMMC-7721 [J]. Chinese Journal of Basic Medicine and Clinical Medicine, 2016, 36 (10): 1335-1340.

[2] Cao Ying, Yu Bin Bin, Zhang Zhong Wei. Bromethamine on Human Hepatocellular Carcinoma SMMC-7721 Cell Proliferation and its Mechanism [J]. Chinese Journal of Hospital Pharmacy, 2016, 36 (19): 1661-1665.

[3] Li Wen Ting, Zhao Feng Ming, Dai Zi Xiang, et al. Hedyotis Diffusa Injection Inhibits the Proliferation and Regulation of Bcl-2 / CytC Signaling Pathway in Human Hepatocellular Carcinoma Cell Line SMMC-7721 [J]. Chinese Traditional Patent Medicine, 2015, 37 (10): 2264-2267.

[4] Liu Z, Dai H, Jia G, et al. Insufficient Radiofrequency Ablationaguon Human Hepatoma SMMC7721 Cell Proliferation by Stimulating Vascular Endothelial Growth Factor Overexpression [J]. Oncology Letters, 2015, 9 (4): 1893-1896.

[5] Wang Feng, Yu Xiao Lan, Xia Ji Yi, et al. Effects of LY294002 on Proliferation and Apoptosis of Human Hepatocellular Carcinoma Cell Line SMMC-7721 [J]. Shandong Medical Journal, 2016, 56 (41): $1-5$.

[6] Wang Xiaofei, Sun Hongfei, Chen Yan Ke, et al. Construction of MicroRNA-17 Family Eukaryotic Expression Vector and its Effect on Proliferation of Human Hepatocellular Carcinoma Cell Line SMMC-7721 [J] Journal of Shanxi Medical University, 2015, 46 (6): 523 (in Chinese with English abstract) [J]. 
Journal of Shanxi Medical University, 2015, 46 (6): $523-527$.

[7] Feng Sun, Bin Zheng. Effect of $\mathrm{Cx} 43$ Gene on Proliferation and Migration of Human Hepatocellular Carcinoma Cell Line SMMC-7721 [J]. Journal of Practical Oncology, 2015, 30(6):527-532. (in Chinese)

[8] Guilan Shi, Lin Huang, Haiyan Qing. Inhibitory Effect of Quercetin on Growth and Apoptosis of Human Hepatoma SMMC-7721 Cells [J]. Chinese Journal of Laboratory Diagnosis, 2015, 13(1):17-19. (in Chinese)
[9] Hanchen Qiu, Yuewen Sun, Shunren Luo. Effects of Saponins from Schizocapsa Plantaginea Hance on Proliferation, Migration and Apoptosis of Human Hepatocellular Carcinoma Cells and its Toxicity to Normal Hepatocytes [J]. Shandong Medical Journal, 2017, 57(15):1-4. (in Chinese)

[10]Li X, Song Z, Zhong H, et al. [Effect of depsides salts from Salvia miltiorrhiza on human hepatoma cell line SMMC-7721 subcutaneous xenografts in nude mice].[J]. Journal of Central South University(Medical Science), 2015, 40(2):158. 